\title{
QUANTIFICAÇÃO E CARACTERIZAÇÃO ENERGÉTICA DA MADEIRA E CASCA DE ESPÉCIES DO CERRADO
}

\section{ENERGETIC QUANTIFICATION AND CARACTERIZATION OF WOOD AND BARK OF SPECIES OF "CERRADO"}

\author{
Ailton Teixeira do Vale ${ }^{1}$ Maria Aparecida Mourão Brasil ${ }^{2} \quad$ Alcides Lopes Leão $^{3}$
}

\begin{abstract}
RESUMO
Em uma faixa de cerrado sensu stricto, de 63,56 ha, da Fazenda Água Limpa, de propriedade da Universidade de Brasília-DF, foram delimitadas dez parcelas de 20 × 50 m cada nas quais se identificaram e medam as alturas totais e o diâmetro, tomado a $30 \mathrm{~cm}$ de altura do solo, de todas as árvores com diâmetro igual ou superior a $5 \mathrm{~cm}$. Foram sorteadas para serem derrubadas e pesadas, no máximo três (3) indivíduos por espécie e por classe de diâmetro, em sete classes diametrais pré-estabelecidas $(5-9 ; 9-13 \ldots ., 29-33 \mathrm{~cm})$. Em cada indivíduo, foram coletadas seções transversais a zero (base), 25, 50, 75 e 100\% (topo) da altura do tronco, partindo da base, embaladas em sacos plásticos e levadas para laboratório, para obtenção da massa específica básica, do poder calorífico superior e dos teores de cinzas, materiais volátil e carbono fixo da madeira e da casca. Foram identificadas 47 espécies. A biomassa seca do povoamento apresentou $71 \%$ de madeira e $29 \%$ de casca, sendo a árvore formada, em média, de $53 \%$ de ramos e $47 \%$ de tronco. A produção média de biomassa seca total para a área foi de $12,38 \mathrm{t} / \mathrm{ha}$, com variações individuais de $0,44 \mathrm{~kg} / \mathrm{ha}$ (Symplocos rhaminifolia, com um indivíduo/ha) a 2.886,04 kg/ha (Sclerolobium paniculatum, com 46 indivíduos/ha). A produção média por árvore foi de $18,39 \mathrm{~kg}$. A massa específica básica da madeira variou de $0,20 \mathrm{~g} / \mathrm{cm}^{3}$ a $0,78 \mathrm{~g} / \mathrm{cm}^{3}$ e a da casca de $0,17 \mathrm{~g} / \mathrm{cm}^{3}$ a $0,67 \mathrm{~g} / \mathrm{cm}^{3}$. O poder calorífico superior variou de $4.516 \mathrm{kcal} / \mathrm{kg}$ a $4.989 \mathrm{kcal} / \mathrm{kg}$, com média de $4.763 \mathrm{kcal} / \mathrm{kg}$, enquanto o da casca variou de $4.187 \mathrm{kcal} / \mathrm{kg}$ a $5.738 \mathrm{kcal} / \mathrm{kg}$. O teor de carbono fixo médio foi de $20,73 \%$ para a madeira e de $25,19 \%$ para a casca. Vochysia thysoidea destacou-se pela grande produção energética (392,49 Mcal/árvore, 20 árvores/ha e $7.849,80 \mathrm{Mcal} / \mathrm{ha}$ ) em função da alta produção de biomassa tanto individual quanto por área, porém com características físicas da madeira inferiores (massa específica baixa $-0,49 \mathrm{~g} / \mathrm{cm}^{3}$ e poder calorífico abaixo da média para a área $-4.713 \mathrm{kcal} / \mathrm{kg}$ ). Acosmium dasycargpum por sua vez apresentou boas características da madeira (alto poder calorífico - $4.989 \mathrm{kcal} / \mathrm{kg}$, alta massa específica $-0,74 \mathrm{~g} / \mathrm{cm}^{3}$ ), mas com baixa produção energética (76,03 Mcal/árvore, 1 árvore/ha e 76,03 Mcal/ha) em função da baixa produção de biomassa. Houve, no entanto, espécies com boas características da madeira e com alta produção de biomassa, individual e/ou por área. São elas: Sclerolobium paniculatum (305,72 Mcal/árvore, 46 árvores/ha, $14.063,12 \mathrm{Mcal} / \mathrm{ha}, 0,72 \mathrm{~g} / \mathrm{cm}^{3}$ e $4849 \mathrm{kcal} / \mathrm{kg}$ ), Dalbergia miscolobium (80,26 Mcal/árvore, 84 árvores/ha, $6.741,84 \mathrm{Mcal} / \mathrm{ha}, 0,77 \mathrm{~g} / \mathrm{cm}^{3}$ e $4896 \mathrm{kcal} / \mathrm{kg}$ ) e Pterodon pubescens $(473,69 \mathrm{Mcal} / \mathrm{árvore}, 14$ árvores/ha, $6.631,66 \mathrm{Mcal} / \mathrm{ha}, 0,73 \mathrm{~g} / \mathrm{cm}^{3}$ e $4953 \mathrm{kcal} / \mathrm{kg}$ ). Essas espécies foram responsáveis por $45,85 \%$ de toda energia disponibilizada na forma de calor, ou seja, $27.437 \mathrm{Mcal} / \mathrm{ha}$.
\end{abstract}

Palavras-chave: produção de energia, biomassa, cerrado.

\section{ABSTRACT}

The purpose of this work was to study the production of energy in an area of sensu stricto cerrado located on the Água Limpa Farm, University of Brasilia - Brazil. Aerial biomass and the characteristics of the wood and bark of the species found in the total area of 63.56 ha were determined. In ten $20 \mathrm{~m} \times 50 \mathrm{~m}$ plots, the total height and diameter, of all the trees whose diameters were either equal to or greater than 5 $\mathrm{cm}$, at $30 \mathrm{~cm}$ from the ground, were measured. Seven diameter classes were pre-determined. Then, three

1. Engenheiro Florestal, Dr., Professor Adjunto do Departamento de Engenharia Florestal, Faculdade de Tecnologia, Universidade de Brasília, CEP 70910-900, Brasília (DF). atvale@unb.br

2. Engenheira Agrônoma, Dr $^{\mathrm{a}}$, Professora Titular aposentada do Departamento de Recursos Naturais, Faculdade de Ciências Agronômicas, Universidade Estadual de São Paulo, CEP 18603-970, Botucatu (SP). mambrasil@laser.com.br

3. Engenheiro Agrônomo, Dr., Professor Livre Docente do Departamento de Recursos Naturais, Faculdade de Ciências Agronômicas, Universidade Estadual de São Paulo, CEP 18603-970, Botucatu (SP). alcidesleao@fca.unep.br 
trees of each species were cut down at random and classified according to its diameter class. The green biomass of the stem and branches of each species was measured in the field. In laboratory, humidity, the wood/bark ratio, specific gravity, heat combustion, the content of fixed carbon, volatile material and ash of the wood and bark were obtained from cross-sections. It was also possible to estimate the dry mass of the wood and branches per tree, species and hectare as well as the amount of heat generated by this cerrado community. Forty-seven species were identified. The dry biomass of the community comprised $71 \%$ of wood and $29 \%$ of bark and trees had an average $53 \%$ of branches and $47 \%$ of stem. The mean production of total dry biomass in the area was $12.38 \mathrm{t} / \mathrm{ha}$. Mean tree production was $18.39 \mathrm{~kg}$. The specific gravity of the wood ranged from $0.2 \mathrm{~g} / \mathrm{cm}^{3}$ to $0.78 \mathrm{~g} / \mathrm{cm}^{3}$, and the bark from $0.17 \mathrm{~g} / \mathrm{cm}^{3}$ to $0.67 \mathrm{~g} / \mathrm{cm}^{3}$. The heat combustion of the woods varied from $4,516 \mathrm{kcal} / \mathrm{kg}$ to $4,989 \mathrm{kcal} / \mathrm{kg}$, whereas that of the bark ranged from $17,526 \mathrm{~kJ} / \mathrm{kg}$ to $24,019 \mathrm{~kJ} / \mathrm{kg}$. The mean heat combustion of the wood was $19,937 \mathrm{~kJ} / \mathrm{kg}$. The mean fixed carbon content was $20.73 \%$ in the wood and $25.19 \%$ in the bark. Vochysia thyrsoidea presented a great energy production $(1,643 \mathrm{MJ} /$ tree, 20 trees/ha e 32,859.00 MJ/ha) due to a great biomass production, but with specific gravity low $\left(0.49 \mathrm{~g} / \mathrm{cm}^{3}\right)$ and heat combustion low $(19,728 \mathrm{~kJ} / \mathrm{kg})$. Acosmium dasycarpum showing wood with good characteristics (heat combustion - 20,883 $\mathrm{kJ} / \mathrm{kg}$, specific gravity $-0,74 \mathrm{~g} / \mathrm{cm}^{3}$ ) did not outstand in the community due to their energy production $(318.26 \mathrm{MJ} / \mathrm{tree}, 1$ tree/ha e $318.26 \mathrm{MJ} / \mathrm{ha}$ ) because their biomass production was low. Nonetheless, there were species that presented wood with good characteristics and high dry biomass production: Sclerolobium paniculatum $(1,279 \mathrm{MJ} /$ trees, 46 trees/ha, 58,834 MJ/ha, $0,72 \mathrm{~g} / \mathrm{cm}^{3}$ e 20,298 kJ/kg), Dalbergia miscolobium (336 MJ/tree, 84 trees $/ \mathrm{ha}, 28,224 \mathrm{MJ} / \mathrm{ha}, 0,77 \mathrm{~g} / \mathrm{cm}^{3}$ e $20,495 \mathrm{~kJ} / \mathrm{kg}$ ) e Pterodon pubescens (1,983 MJ/tree, 14 trees/ha, 27,760 MJ/ha, 0,73 g/ $\mathrm{cm}^{3}$ e 20,733 kJ/kg).

Keywords: energy production, biomass, "cerrado".

\section{INTRODUÇÃO}

O modelo energético brasileiro tem, no petróleo, uma das principais fontes primárias de energia fóssil, portanto, finito. Por outro lado, o Brasil é um país rico em possibilidades energéticas alternativas, tais como: a energia solar, a eólica, a geotérmica, a das marés e a nuclear que devem ser estudas e exploradas para suprir a demanda futura de energia. Entre as alternativas renováveis existentes, em relação aos combustíveis fósseis, a biomassa tem despertado maior interesse.

A biomassa, no Brasil, é constituída em grande parte pela madeira, onde o uso se divide em produção de carvão vegetal (carbonização) e consumo direto (combustão). No consumo direto da madeira, como fonte de energia, destaca-se o uso para cocção de alimentos nas residências, principalmente no meio rural. As principais fontes de madeira para suprimento energético encontram-se nas matas nativas, principalmente Cerrado e Mata Atlântica.

O cerrado ocupa praticamente um quarto do território brasileiro, é fonte da lenha nativa para a geração de calor e, talvez, o maior fornecedor de combustível para o cozimento no meio rural o que o situa como um dos biomas de grande importância social.

Pouco se conhece das espécies do cerrado do ponto de vista energético, uma vez que são utilizadas de maneira indiscriminada. Nesse contexto, o presente trabalho teve como objetivos quantificar a energia disponível na forma de calor e caracterizar a madeira e a casca de espécies de cerrado que ocorrem no Distrito Federal.

\section{REVISÃO BIBLIOGRÁFICA}

Dentre as alternativas estudadas para suprir a demanda ener gética, a biomassa é o combustível renovável que tem despertado maior interesse. Segundo Grassi e Palz (1994), "a produção sustentada de biomassa, que é a fonte mais versátil de energia renovável, apresenta a possibilidade de prover, de forma permanente, grandes quantidades de combustíveis gasosos, líquidos e eletricidade".

Segundo Hall (1991), a biomassa representa cerca de $14 \%$ da energia consumida no mundo e, em média, 35\% do consumo total de países em desenvolvimento.

No Brasil, a biomassa formada pela cana-de-açúcar e pela madeira representou 19,39\% de todos os 
energéticos primários consumidos em 1999 (Brasil, 2000). Desse total, 9,12\% foram compostos pela lenha, ou seja, $6,9 \times 10^{7}$ toneladas de madeira foram utilizadas para geração de calor. Do total da lenha consumida, $2,5 \times 10^{7}$ toneladas o foram em forma indireta, principalmente na fabrica ção de carvão vegetal, e $4,4 \times 10^{7}$ toneladas, em forma direta. Do total consumido em forma direta, $2,1 \times 10^{7}$ toneladas foram queimadas em residências, para cocção de alimentos.

É importante observar que não pesa somente o aspecto quantitativo do uso da madeira como energia, mas também o aspecto econômico, ligado à utilização energética pelas indústrias, e, mais importante ainda, o aspecto social, ou seja, a lenha como energético doméstico (Brito e Deglise, 1991), que, segundo Goldemberg (1998), é a fonte de energia dominante nas áreas rurais, e cozinhar é a atividade mais intensiva energeticamente.

No Brasil, a principal fonte de madeira para produção de energia tem sido os ecossistemas naturais, com o cerrado e a mata atlântica ocupando os primeiros lugares. O uso mais intensivo da madeira como energético está concentrado nas regiões Sul, Sudeste e Nordeste. A diminuição desses biomas e a pressão conservacionista, associadas à necessidade anual de mais madeira para energia, têm levado a uma crescente dificuldade para a obtenção desse recurso com base em florestas nativas (Brito e Deglise, 1991).

Mata (2000) enfatizou a necessidade de estudos sistemáticos sobre a evolução do consumo "que resultem em diagnósticos adequados sobre o uso e a conservação da biomassa energética, em especial de lenha, para muitas comunidades onde, geralmente, se observa enorme esfor ço associado à obtenção da mesma".

Arouca (1983), afirma que, em conseqüência à grande influência que o consumo de lenha acarreta na estrutura de energia no setor residencial, deve-se conhecer melhor o seu consumo, rendimento e conteúdo calórico.

Com estudos que visem a diagnosticar o consumo e, ao mesmo tempo, conhecer o as características da madeira que compõe a biomassa de um povoamento, será possível propor, como sugere Oliveira et al. (1998), a busca de alternativas para o uso sustentado da vegeta ção nativa.

\section{MATERIAL E MÉTODOS}

\section{Localização da área de estudo}

A coleta de dados de campo foi conduzida em área de vegetação do tipo cerrado sensu stricto na Fazenda Água Limpa (FAL), de propriedade da Universidade de Brasília (UnB), Distrito Federal. A FAL está localizada em altitude de 1.100 metros a $15^{\circ} 56^{\prime} 14^{\prime}$ 'S e $47^{\circ} 46^{\prime} 08^{\prime}$ ' $\mathrm{W}$, tem sido utilizada como fazenda experimental e reserva ecológica e compreende área aproximada de 4.000 ha.

\section{Os solos}

Predominam, na Fazenda Água Limpa, cobrindo aproximadamente 70\% da área, o latossolo vermelho-escuro e o latossolo vermelho-amarelo, e ocorrem também em pequena quantidade, cambissolo e solos hidromórficos (EMBRAPA, 1978). Segundo Haridasan (1990), esses solos, em geral, s ão distróficos e muito ácidos, com elevados níveis de alumínio trocável. Na área da coleta o solo é do tipo latossolo vermelho-escuro (Silva, 1999).

\section{O clima}

Pela classificação de Köppen e de acordo com Nimer (1989) citado por Silva (1999), o clima é do tipo Aw, com temperatura média anual de, aproximadamente, $20,4^{\circ} \mathrm{C}$, e valores de máxima e mínima de, respectivamente, $28,5^{\circ} \mathrm{C}$ e $12,0^{\circ} \mathrm{C}$. Segundo o mesmo autor, a média anual de precipitação é de $1.500 \mathrm{~mm}$, com verões úmidos e invernos secos, com início das chuvas em setembro, pequenas ocorrências em junho e agosto, atingindo o máximo em novembro.

\section{A vegetação}


Distribuem-se nos 4.000 ha da Fazenda Água Limpa 28,20\% de campo limpo, 4,40\% de campos e murunduns, $20,10 \%$ de campo sujo, $36,50 \%$ de cerrado sensu stricto, $0,20 \%$ de cerradão e $9,90 \%$ de matas de galerias e outras formações incipientes (Furley e Ratter, 1990). A área amostrada faz parte do cerrado sensu stricto.

\section{Amostragem}

A área de 63,54 ha foi dividida em parcelas iguais de $20 \mathrm{~m}$ x $50 \mathrm{~m}$ dentre as quais sortearam-se dez parcelas, totalizando uma amostra de $1,57 \%$ da área. Procedimento semelhante foi utilizado por Silva (1990) que amostrou $1,5 \%$ da área estudada, para estudos de compartilhamento de nutrientes. Cada parcela foi delimitada e todas as árvores com diâmetro igual ou superior a $5 \mathrm{~cm}$ medido a $30 \mathrm{~cm}$ de altura do solo foram numeradas e identificadas botanicamente, anotando-se os diâmetros e as alturas totais.

As árvores devidamente identificadas foram distribuídas nas classes de diâmetro de 5-9; 9-13; 13 $17 ; 17-21 ; 21-25 ; 25-29$ e 29-33cm. Após essa classificação, sortearam-se, ao acaso, três indivíduos por classe diamétrica e por espécie que foram cortados para a pesagem do tronco e dos ramos, separadamente, no campo, e a retirada de amostras da madeira e da casca.

$\mathrm{O}$ tronco e os ramos foram pesados separadamente. Ambas as massas foram obtidas com casca. A seguir, foram recolhidas duas amostras de seções transversais (discos) com aproximadamente $2,50 \mathrm{~cm}$ de espessura, ao longo do tronco nas posições correspondentes a $0,25 \%, 50 \%, 75 \%$ e $100 \%$ da altura do tronco. Foi retirada, também, amostra na base de cada ramo. Essas amostras foram encaminhadas para o laboratório onde foi determinada a relação em massa entre a madeira e a casca, em base úmida e também os seus teores de umidade, segundo Vital (1997). Com essa rela ção foi possível estimar a biomassa de tronco, de ramos e de casca, em base úmida, no campo. Com os valores estimados de biomassa úmida no campo e os teores de umidade obtidos, foi possível estimar a massa seca no campo.

Do disco obtido a $25 \%$ da altura do tronco, partindo da base, foi retirada uma amostra em for ma de cunha para determinação da massa específica básica, segundo Foelkel et al. (1971) e Vital (1984). Na determinação da massa específica básica da casca, utilizou-se uma amostra composta, com base em discos de todas as alturas.

Os discos destinados às análises imediatas e ao poder calorífico foram separados em madeira e casca. Cada fração foi misturada numa amostra composta por espécie. As amostras compostas, após serem moídas, foram classificadas em peneiras, obtendo-se a fração retida entre 40 e 60 mesh que foi utilizada para a determinação do poder calorífico superior, segundo a norma ABNT NBR 8633/84 utilizando-se da bomba calorimétrica PARR 1201, e a fração abaixo de 60 mesh, para a análise imediata, segundo a norma ASTM D-1762/64.

A quantidade disponibilizada de calor para a espécie foi obtida pelo produto da massa seca de madeira com o respectivo poder calorífico para cada espécie. De maneira semelhante, obteve-se a quantidade de calor originada pela casca. A Figura 1 apresenta o organograma das atividades de coleta de dados. 


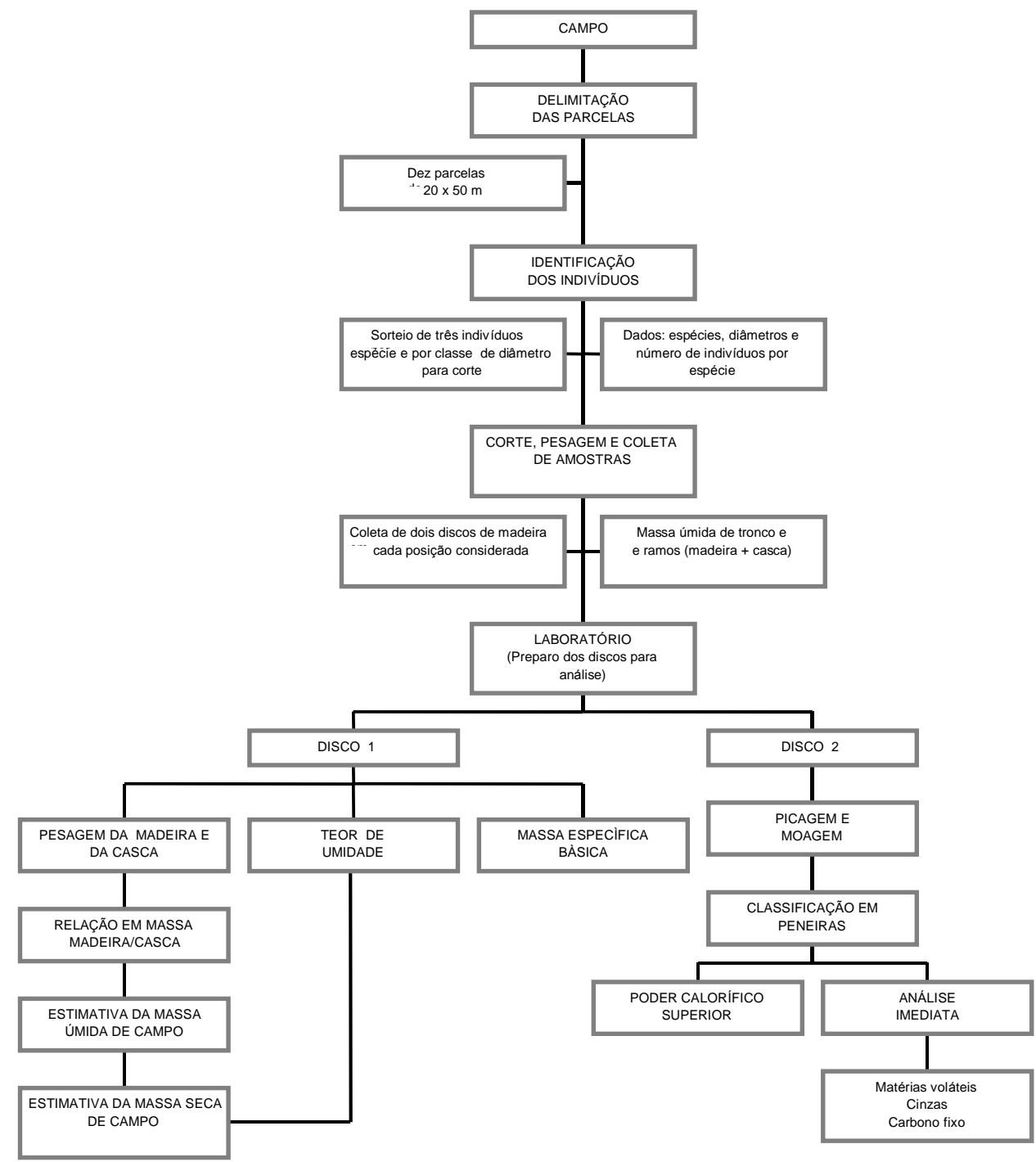

FIGURA 1: Organograma das atividades desenvolvidas durante o trabalho.

\section{RESULTADOS E DISCUSSÃO}

A Tabela 1 apresenta os dados de massa seca e quantidade de calor por indivíduo, bem como o número de indivíduos encontrados por hectare e, a Tabela 2, as características das madeiras e cascas das espécies.

\section{Densidade básica da madeira e da casca}

O uso de madeiras com baixa densidade para a produ ção direta de energia na forma de calor implica em uma queima rápida e numa menor produção de energia por unidade de volume ao contrário de madeiras com maiores densidades. Porém, densidade muita elevada implica em dificuldade de iniciar a queima do material. Para a queima direta na cocção de alimentos sugere-se a faixa intermediária entre madeiras médias e madeiras duras, variando a densidade básica de 0,65 a $0,80 \mathrm{~g} / \mathrm{cm}^{3}$, esperando, com isso, facilitar o início da queima.

A densidade básica da madeira das espécies do cerrado, como pode ser observado, na Tabela 2 , apresenta uma variação de $0,20 \mathrm{~g} / \mathrm{cm}^{3}$ a $0,78 \mathrm{~g} / \mathrm{cm}^{3}$, enquanto a densidade básica da casca variou de 0,17 $\mathrm{g} / \mathrm{cm}^{3}$ a $0,67 \mathrm{~g} / \mathrm{cm}^{3}$. Richter e Burger (1991), afirmam que a variação da densidade básica da madeira é de 0,13 a $1,4 \mathrm{~g} / \mathrm{cm}^{3}$. O cerrado, em estudo, não apresenou madeiras extremamente duras, tendo apenas 
TABELA 1: Número de indivíduos por ha (NI), massa seca média e quantidade de calor médio por indivíduo, para madeira e casca das espécies do cerrado

\begin{tabular}{|c|c|c|c|c|c|c|c|c|}
\hline \multirow{3}{*}{ Espécies } & \multirow{3}{*}{ NI } & \multirow{2}{*}{\multicolumn{3}{|c|}{$\begin{array}{l}\text { Massa seca } \\
\text { kg/árvore }\end{array}$}} & \multicolumn{4}{|c|}{ Quantidade de calor } \\
\hline & & & & & \multicolumn{3}{|c|}{ Mcal/árvore } & \multirow{2}{*}{$\begin{array}{c}\text { Mcal/ha } \\
\text { Total }\end{array}$} \\
\hline & & Madeira & Casca & Total & Madeira & Casca & Total & \\
\hline 01-Acosmium dasycarpum & 1 & 10,91 & 4,25 & 15,16 & 54,44 & 21,59 & 76,03 & 76,03 \\
\hline 02-Aspidosperma macrocarpon & 5 & 5,58 & 2,93 & 8,51 & 26,94 & 15,51 & 42,44 & 212,2 \\
\hline 03-Aspidosperma tomentosum & 11 & 3,60 & 1,94 & 5,54 & 17,51 & 9,15 & 26,66 & 293,26 \\
\hline 04-Byrsonima coccolobifolia & 2 & 2,46 & 1,54 & 4,00 & 11,92 & 7,77 & 19,69 & 39,38 \\
\hline 05-Byrsonima crassa & 11 & 1,31 & 0,82 & 2,13 & 6,26 & 3,98 & 10,24 & 112,64 \\
\hline 06-Byrsonima verbascifolia & 4 & 1,25 & 0,91 & 2,16 & 5,96 & 4,47 & 10,43 & 41,72 \\
\hline 07-Blepharocalix salicifolius & 12 & 23,43 & 11,47 & 34,90 & 98,32 & 51,56 & 149,88 & 1798,56 \\
\hline 08-Caryocar brasiliense & 29 & 14,54 & 9,88 & 24,04 & 69,25 & 46,33 & 115,58 & 3351,82 \\
\hline 09-Connarus suberosus & 7 & 0,46 & 0,38 & 0,84 & 2,21 & 1,98 & 4,19 & 29,33 \\
\hline 10-Dalbergia miscolobium) & 84 & 12,03 & 4,19 & 16,22 & 58,91 & 21,35 & 80,26 & 6741,84 \\
\hline 11-Dimorphandra mollis & 1 & 6,37 & 2,72 & 9,09 & 31,47 & 14,09 & 45,56 & 45,56 \\
\hline 12-Enterolobium gummiferum & 1 & 3,92 & 1,76 & 5,68 & 18,57 & 9,22 & 27,79 & 27,79 \\
\hline 13-Eremanthus glomerulatus & 16 & 0,33 & 0,17 & 0,51 & 1,56 & 0,84 & 2,41 & 38,56 \\
\hline 14-Eriotheca gracilipes & 2 & 1,98 & 1,65 & 3,63 & 9,04 & 8,02 & 17,06 & 34,12 \\
\hline 15-Eriotheca pubescens & 10 & 5,40 & 4,25 & 9,65 & 24,65 & 21,19 & 45,84 & 458,4 \\
\hline 16-Erythroxylum deciduum & 1 & 0,46 & 0,42 & 0,88 & 2,13 & 2,09 & 4,23 & 4,23 \\
\hline 17-Erythroxylum suberosum & 2 & 0,55 & 0,51 & 1,06 & 2,50 & 2,56 & 5,07 & 10,14 \\
\hline 18-Erythroxylum to & 3 & 0,39 & 0,59 & 0,98 & 1,92 & 3,04 & 4,96 & 14,88 \\
\hline 19-Guapira & 10 & 3,34 & 0,64 & 3,98 & 18,16 & 3,27 & 21,43 & 214,3 \\
\hline 20-Hymenaea stigonocarpa & 3 & 57,66 & 42,43 & 100,09 & 279,73 & 203,47 & 483,19 & 1449,57 \\
\hline 21-Kielmeyera coriacea & 21 & 0,67 & 0,67 & 1,34 & 3,18 & 3,69 & 6,87 & 144,27 \\
\hline 22-Kielmeyera speciosa & 12 & 1,55 & 1,60 & 3,15 & 7,57 & 8,92 & 16,49 & 197,88 \\
\hline 23-Lafoensia pacari & 1 & 8,29 & 5,96 & 14,25 & 39,69 & 29,72 & 69,42 & 69,42 \\
\hline 24-Miconia ferruginata & 8 & 3,19 & 1,00 & 4,19 & 15,24 & 4,57 & 19,81 & 158,48 \\
\hline 25-Miconia pohliana & 35 & 2,93 & 1,50 & 4,43 & 13,55 & 6,88 & 20,43 & 715,05 \\
\hline 26-Myrsine guianensis & 3 & 0,85 & 0,69 & 1,54 & 4,00 & 3,53 & 7,53 & 22,59 \\
\hline 27-Ouratea hexasperma & 114 & 3,23 & 3,39 & 6,62 & 15,91 & 17,73 & 33,64 & 3834,96 \\
\hline 28-Palicourea rigida & 32 & 0,66 & 0,46 & 1,12 & 3,10 & 2,34 & 5,44 & 174,08 \\
\hline 29-Pouteria ramiflora & 8 & 8,35 & 4,35 & 12,70 & 39,91 & 22,07 & 61,97 & 495,76 \\
\hline 30-Piptocarpha rotundifolia & 3 & 1,31 & 1,03 & 2,34 & 6,22 & 5,11 & 11,32 & 33,96 \\
\hline 31-Psidium warmingianum & 1 & 1,17 & 1,54 & 2,71 & 5,56 & 7,59 & 13,15 & 13,15 \\
\hline 32-Pterodon pubescens & 14 & 76,86 & 18,91 & 95,77 & 380,69 & 93,01 & 473,69 & 6631,66 \\
\hline 33-Qualea grandiflora & 34 & 17,75 & 6,69 & 24,44 & 84,07 & 31,60 & 115,67 & 3932,78 \\
\hline 34-Qualea multiflora & 23 & 3,88 & 1,06 & 4,94 & 18,34 & 4,58 & 22,92 & 527,16 \\
\hline 35-Qualea $p$ & 35 & 14,97 & 7,01 & 21,98 & 70,52 & 32,70 & 103,21 & 3612,35 \\
\hline 36-Rourea induta & 2 & 84 & 1,20 & & 8,59 & 5,98 & 14,57 & 29,14 \\
\hline 37-Scheflera ma & 25 & & 3,35 & & 31,66 & 16,26 & 47,92 & 1198 \\
\hline 38-Sclerolobium paniculatum & 46 & & 13,90 & & 236,84 & 68,89 & 305,72 & 14063,12 \\
\hline 39-Strychnos pseudoquina & 1 & & 5,84 & 27,66 & 103,78 & 33,52 & 137,29 & 137,29 \\
\hline 40-Stryphnodendron adstringens & 5 & 13,58 & 7,18 & 20,76 & 65,40 & 34,96 & 100,36 & 501,80 \\
\hline 41-Styrax ferrugineus & 5 & 6,31 & 3,05 & 9,36 & 30,01 & 15,84 & 45,84 & 229,20 \\
\hline 42-Symplocos rhamnifolia & 1 & 0,20 & 0,24 & 0,44 & & 1,12 & 1,12 & 1,12 \\
\hline 43-Tabebuia ochracea & 1 & 2,01 & 2,17 & 4,18 & 9,57 & 10,89 & 20,45 & 20,45 \\
\hline 44-Tabebuia serratifolia & 1 & 4,70 & 2,19 & 6,89 & 22,67 & 10,59 & 33,26 & 33,26 \\
\hline 45-Vochysia elliptica & 6 & 4,57 & 2,62 & 7,19 & 21,65 & 12,13 & 33,78 & 202,68 \\
\hline 46-Vochysia rufa & 1 & 1,19 & 0,71 & 1,90 & 5,57 & 3,14 & 8,71 & 8,71 \\
\hline 47-Vochysia thyrsoidea & 20 & 60,75 & 22,36 & 83,11 & 286,33 & 106,15 & 392,49 & 7849,8 \\
\hline
\end{tabular}

Em que: $\mathrm{NI}=$ número de indivíduos. 
TABELA 2: Densidade básica (Db), poder calorífico superior (PCS) e teor de material volátil (MV), de cinza (CZ) e de carbono fixo (CF) da madeira e da casca para as espécies do cerrado da FAL/UnB/DF.

\begin{tabular}{|c|c|c|c|c|c|c|c|c|c|c|}
\hline \multirow[b]{2}{*}{ Espécies } & \multicolumn{5}{|c|}{ Madeira } & \multicolumn{5}{|c|}{ Casca } \\
\hline & \begin{tabular}{|c|}
$\mathrm{Db}$ \\
$\mathrm{g} / \mathrm{cm}^{3}$
\end{tabular} & \begin{tabular}{|c|}
$\mathrm{PCS}$ \\
$\mathrm{kcal} / \mathrm{kg}$
\end{tabular} & $\begin{array}{c}\text { MV } \\
\%\end{array}$ & $\begin{array}{c}\mathrm{CZ} \\
\%\end{array}$ & $\begin{array}{c}\mathrm{CF} \\
\%\end{array}$ & \begin{tabular}{|c|}
$\mathrm{Db}$ \\
$\mathrm{g} / \mathrm{cm}^{3}$
\end{tabular} & $\begin{array}{c}\mathrm{PCS} \\
\mathrm{kcal} / \mathrm{kg}\end{array}$ & $\begin{array}{c}\mathrm{MV} \\
\%\end{array}$ & $\begin{array}{c}\mathrm{CZ} \\
\%\end{array}$ & $\begin{array}{c}\mathrm{CF} \\
\%\end{array}$ \\
\hline Acosmium dasycarpum & 0,74 & 4989,60 & 78,59 & 0,41 & 21,00 & 0,54 & 5081,00 & 71,43 & 3,55 & 25,02 \\
\hline Aspidosperma macrocarpon & 0,61 & 4827,50 & 78,96 & 0,27 & 20,77 & 0,46 & 5292,20 & 75,54 & 1,16 & 23,30 \\
\hline Aspidosperma to mentosum & 0,58 & 4863,30 & 80,32 & 0,35 & 19,33 & 0,30 & 4717,40 & 74,82 & 2,39 & 22,79 \\
\hline Byrsonima coccolobifolia & 0,59 & 4844,50 & 77,40 & 0,94 & 21,66 & 0,50 & 5047,40 & 69,26 & 1,66 & 29,08 \\
\hline Byrsonima crassa & 0,56 & 4781,40 & 75,38 & 1,09 & 23,53 & 0,42 & 4849,90 & 73,47 & 0,97 & 25,56 \\
\hline Byrsonima verbascifolia & 0,48 & 4771,50 & 74,62 & 1,24 & 24,14 & 0,46 & 4909,40 & 68,80 & 1,63 & 29,57 \\
\hline Blepharocalix salicifolius & 0,46 & 4516,40 & 78,87 & 0,68 & 20,45 & 0,50 & 4805,00 & 73,52 & 2,32 & 24,16 \\
\hline Caryocar brasiliense & 0,61 & 4839,10 & 80,13 & 0,37 & 19,50 & 0,41 & 4761,80 & 75,72 & 0,65 & 23,63 \\
\hline Connarus suberosus & 0,52 & 4813,50 & 74,93 & 0,62 & 24,45 & 0,41 & 5206,20 & 67,18 & 0,68 & 32,14 \\
\hline Dalbergia miscolobium & 0,77 & 4896,90 & 78,29 & 0,26 & 21,45 & 0,43 & 5096,70 & 72,51 & 0,79 & 26,70 \\
\hline Dimorphandra mollis & 0,70 & 4940,70 & 80,24 & 0,17 & 19,59 & 0,46 & 5178,30 & 71,81 & 0,40 & 27,79 \\
\hline Enterolobium gummiferum & 0,62 & 4737,80 & 80,98 & 0,14 & 18,88 & 0,46 & 5239,90 & 70,02 & 0,75 & 29,23 \\
\hline Eremanthus glomerulatus & 0,57 & 4738,80 & 80,50 & 0,66 & 18,84 & 0,25 & 4967,80 & 75,05 & 1,33 & 23,62 \\
\hline Eriotheca grc & 0,36 & 4565,90 & 74,76 & 1,91 & 23,33 & 0,35 & 4857,70 & 75,37 & 1,31 & 23,32 \\
\hline & 0,38 & 4565,50 & 79,08 & 1,00 & 19,92 & 0,44 & 4985,40 & 76,26 & 1,05 & 22,69 \\
\hline & 0,52 & 4638,00 & 76,75 & 2,15 & 21,10 & 0,41 & 4985,60 & 73,86 & 1,34 & 24,8 \\
\hline Ery & 0,62 & 4549,90 & 76,17 & 2,73 & 21,10 & & 5028,00 & 73,59 & 1,59 & 24,82 \\
\hline & 0,54 & 4931,90 & 75,83 & 0,53 & 23,64 & 0,42 & 5148,10 & 68,76 & 1,31 & 29,93 \\
\hline & 0,47 & 4622,10 & 75,59 & 1,40 & 23,01 & 0,39 & 4187,70 & 73,99 & 2,07 & 23,94 \\
\hline & 0,78 & 4851,30 & 77,37 & 1,08 & 21,55 & 0,67 & 4795,40 & 76,58 & 0,76 & 22,66 \\
\hline & 0,46 & 4747,40 & 79,27 & 0,31 & 20,42 & 0,29 & 5502,00 & 72,26 & 1,03 & 26,71 \\
\hline & 0,58 & 4882,70 & 79,08 & 0,57 & 20,35 & 0,48 & 5576,80 & 73,89 & 0,81 & 25,30 \\
\hline & 0,74 & 4788,00 & 74,67 & 0,57 & 24,76 & 0,67 & 4987,10 & 68,66 & 0,73 & 30,61 \\
\hline inata & 0,65 & 4777,00 & 75,49 & 0,39 & 24,12 & 0,39 & 4570,30 & 65,27 & 4,25 & 30,48 \\
\hline & 0,57 & 4626,20 & 75,42 & 0,40 & 24,18 & 0,53 & 4586,10 & 72,24 & 2,35 & 25,41 \\
\hline & 0,52 & 4700,90 & 79,90 & 0,53 & 19,57 & 0,42 & 5116,50 & 69,83 & 1,89 & 28,28 \\
\hline & 0,50 & 4926,30 & 76,94 & 0,90 & 22,16 & 0,50 & 5229,50 & 65,2 & 1,09 & 33,71 \\
\hline Palico & 0,43 & 4695,20 & 79,80 & 1,10 & 19,10 & 0,37 & 5096,40 & 73,68 & 2,48 & 23,84 \\
\hline Pout & 0,70 & 4779,10 & 78,28 & 0,57 & 21,15 & 0,41 & 5073,10 & 71,63 & 1,02 & 27,35 \\
\hline Pipt & 0,42 & 4744,30 & 76,59 & 0,90 & 22,51 & 0,33 & 4959,10 & 72,93 & 1,58 & 25,49 \\
\hline & 0,20 & 4752,10 & 76,60 & 1,01 & 22,39 & 0,49 & 4928,40 & 67,78 & 0,43 & 31,79 \\
\hline & 0,73 & 4953,00 & 80,42 & 0,24 & 19,34 & 0,41 & 4918,30 & 74,68 & 1,01 & 24,31 \\
\hline & 0,69 & 4736,40 & 76,37 & 0,36 & 23,27 & 0,44 & 4722,90 & 71,87 & 2,14 & 25,99 \\
\hline & 0,66 & 4725,90 & 75,63 & 0,56 & 23,81 & 0,37 & 4323,20 & 75,38 & 2,14 & 22,48 \\
\hline Quc & 0,69 & 4710,50 & 77,07 & 0,81 & 22,12 & 0,50 & 4664,10 & 72,87 & 3,13 & 24,00 \\
\hline Rou & 0,47 & 4667,80 & 79,04 & 1,91 & 19,05 & 0,59 & 4986,30 & 70,63 & 0,79 & 28,58 \\
\hline & 0,68 & 4740,00 & 78,93 & 0,63 & 20,44 & 0,46 & 4854,00 & 74,67 & 2,04 & 23,29 \\
\hline Scl & 0,72 & 4849,20 & 78,61 & 0,39 & 21,00 & 0,56 & 4956,00 & 67,01 & 0,68 & 32,31 \\
\hline & 0,72 & 4756,00 & 79,98 & 0,51 & 19,51 & 0,48 & 5738,90 & 69,81 & 1,38 & 28,81 \\
\hline Stry & 0,55 & 4816,00 & 77,83 & 0,22 & 21,95 & 0,42 & 4869,40 & 66,22 & 1,58 & 32,20 \\
\hline & 0,49 & 4755,40 & 78,94 & 0,60 & 20,46 & 0,47 & 5192,60 & 67,70 & 0,77 & 31,53 \\
\hline Symploco & 0,38 & $*$ & $*$ & $*$ & $*$ & 0,17 & 4682,30 & $*$ & $*$ & $*$ \\
\hline Tabebuia & 0,62 & 4760,30 & 81,20 & 0,42 & 18,38 & 0,42 & 5016,40 & 76,52 & 1,45 & 22,03 \\
\hline Tabebuias & 0,69 & 4823,80 & & 0,84 & 20,66 & 0,40 & & 74,77 & 2,19 & 23,04 \\
\hline Vochysia elliptica & 0,57 & 4736,80 & 80,69 & 1,38 & 17,93 & 0,45 & 4630,10 & 76,27 & 2,31 & 21,42 \\
\hline Vochysia rufa & 0,40 & 4680,20 & 79,12 & 1,44 & 19,44 & 0,35 & 4416,50 & & $*$ & \\
\hline
\end{tabular}


\begin{tabular}{l|lllll|lllll} 
Vochysia thyrsoidea Pohl. & 0,49 & 4713,30 & 80,03 & 0,81 & 19,16 & 0,54 & 4747,40 & 76,95 & 2,78 & 20,27 \\
\hline
\end{tabular} Em que: * = Material insuficiente para determinação.

sete espécies com madeiras consideradas duras $\left(\mathrm{Db}>0,70 \mathrm{~g} / \mathrm{cm}^{3}\right)$. Essas sete espécies são responsáveis por 22,28\% dos indivíduos. Dentre essas sete espécies se destacam três: Sclerolobium paniculatum, Pterodon pubescens e Dalbergia miscolobium que representam $21,39 \%$ dos indivíduos. A maior densidade foi a da espécie Hymenaea stigonocarpa $\left(0,78 \mathrm{~g} / \mathrm{cm}^{3}\right)$.

\section{Análise imediata da madeira e da casca}

Combustíveis com alto índice de carbono fixo devem ter queima mais lenta, implicando maior tempo de residência dentro dos aparelhos de queima, em comparação com outros que tenham menor teor de carbono fixo (Brito e Barrichello (1982).

A queima mais lenta pode ser vantajosa para o cozimento de alimentos, uma vez que os aparelhos de queima no meio rural (fogões) têm eficiência muito baixa na utilização do calor produzido, que, segundo Goldemberg (1998), converteria apenas ao redor de $10 \%$ da energia contida na lenha em energia útil na cocção.

A menor quantidade de matérias voláteis da madeira $(74,62 \%)$ foi detectada em Byrsonima verbascifolia, e o maior teor, de 81,2\%, em Tabebuia ochracea. A casca de Ouratea hexasperma apresentou o menor teor de matérias voláteis (65,2\%), enquanto o maior teor foi de 76,95\%, em Vochysia thyrsoidea.

O valor médio em carbono fixo para a madeira foi de $20,73 \%$, e, para a casca, de $25,19 \%$. Lafoensia pacari destacou-se com o maior valor de carbono fixo na madeira (24,75\%), e Vochysia elliptica, com o menor (17,93\%). Na casca, os valores máximo e mínimo foram de 33,70\% e 20,26\% respectivamente, para Ouratea hexasperma e Vochysia thyrsoidea.

Os teores de matérias voláteis e carbono fixo na madeira estão de acordo com Brito e Barrichello (1982) que preconizaram, em termos gerais, teores de matérias voláteis entre $75 \%$ a $85 \%$ e de carbono fixo entre $15 \%$ a $25 \%$.

Os teores de cinzas e de carbono fixo da casca foram superiores aos da madeira, diferentemente do que ocorreu com matérias voláteis. Resultados semelhantes para os três parâmetros foram encontrados por Brito e Barrichello (1978) em trabalho com cinco espécies de eucaliptos.

A variação dos teores de cinzas da madeira ficou entre $0,15 \%$ (Enterolobium gummiferum) e 2,73\% (Erytroxylum suberosum). Para a casca, a variação foi de 0,41\% (Dimorphandra mollis) a 4,25\% (Miconia ferruginata). Esses teores estão dentro de faixas encontradas por outros autores para outras esp écies, como Castillo (1984), Cunha et al. (1989) e Maraboto et al. (1989) que, trabalhando com 80 espécies de folhosas da região Amazônica, encontraram variação no teor de cinzas da madeira de 0,05\% em Ocotea cymbarum (inhamuí), a 3\%, em Erisma uncinatum (quarubarana). Brito e Barrichello (1978) encontraram, na casca de cinco espécies de eucalipto uma variação de $1,34 \%$ a $6,40 \%$.

Verifica-se pela Tabela 2 que as três espécies, que sobressaíram quanto à densidade e produção de energia: Sclerolobium paniculatum, Pterodon pubescens e Dalbergia miscolobium, possuem valores médios de carbono fixo e baixos valores de cinzas.

O poder calorífico superior da madeira das 47 espécies variou de $4.516 \mathrm{kcal} / \mathrm{kg}$ para Blepharocalix salicifolius a $4.989 \mathrm{kcal} / \mathrm{kg}$ para Acosmium dasycarpum. O valor médio, para o cerrado, foi de $4.763 \mathrm{kcal} / \mathrm{kg}$, superior aos valores preconizados para folhosas $(4500 \mathrm{kcal} / \mathrm{kg})$ e àqueles encontrados para espécies da Amazônia que, segundo Castillo (1984) que trabalhou com vinte espécies da Amazônia peruana, é de $4.751 \mathrm{kcal} / \mathrm{kg}$, com variações de $4.621 \mathrm{kcal} / \mathrm{kg}$ para Micranda spruceana a $4.885 \mathrm{kcal} / \mathrm{kg}$ para Trichilia sexanthera.

O poder calorífico superior da casca variou de $4.187 \mathrm{kcal} / \mathrm{kg}$ (Guapira noxia) a $5.738 \mathrm{kcal} / \mathrm{kg}$ (Strychnus pseudoquina) e, foi, em geral, superior ao da madeira.

Jara (1989) determinou o poder calorífico da casca de 27 espécies, encontrando variação de $3.822 \mathrm{kcal} / \mathrm{kg}$ para Eucalyptus grandis com nove anos de idade a $5.837 \mathrm{kcal} / \mathrm{kg}$ para Paulownia tomentosa 
Quantificação e caracterização energética da madeira e casca de espécies do cerrado

(kiri).

Novamente entre outras espécies, como Dimorphandra mollis, com $4.940 \mathrm{kcal} / \mathrm{kg}$ e Ouratea hexasperma com $4926 \mathrm{kcal} / \mathrm{kg}$; Sclerolobium paniculatum $(4.849 \mathrm{kca} / \mathrm{kg})$, Pterodon pubescens $(4.953$ $\mathrm{kcal} / \mathrm{kg})$ e Dalbergia miscolobium $(4.896 \mathrm{kcal} / \mathrm{kg})$ destacam-se com valores de poder calorífico superior acima da média.

\section{Quantidade de energia na forma de calor}

A quantidade de calor para a madeira variou de 1,6 Mcal/árvore (Eremanthus glomerulatus) a $380 \mathrm{Mcal} / a ́ r v o r e$ (Pterodon pubescens) e, para casca, a variação foi de 1,1 Mcal/árvore (Symplocos rhaminifolia) a 106 Mcal/árvore (Vochysia thyrsoidea). Doze espécies destacaram-se na produção de calor

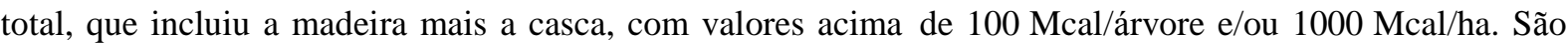
elas: Pterodon pubescens (473 Mcal/árvore e $6.632 \mathrm{Mcal} / \mathrm{ha}$ ), Vochysia thyrsoidea (392 Mcal/árvore e 7.850 $\mathrm{Mcal} / \mathrm{ha}$ ), Sclerolobium paniculatum (306 Mcal/árvore e $14.063 \mathrm{Mcal} / \mathrm{ha}$ ), Hymenaea stigonocarpa (280 Mcal/árvore e $1.450 \mathrm{Mcal} / \mathrm{ha})$, Blepharocalix salicifolius (150 Mcal/árvore e $1.799 \mathrm{Mcal} / \mathrm{ha})$, Strychnus pseudoquina (137 Mcal/árvore), Caryocar brasiliense (115 Mcal/árvore e $3.352 \mathrm{Mcal} / \mathrm{ha})$, Qualea grandiflora (115 Mcal/árvore e $3.933 \mathrm{Mcal} / \mathrm{ha})$, Q. parviflora (103 Mcal/árvore e $3.612 \mathrm{Mcal} / \mathrm{ha}$ ), Stryphnodendron adstrigens (100 Mcal/árvore), Dalbergia miscolobium $(6.742 \mathrm{Mcal} / \mathrm{ha})$ e Ouratea hexasperma $(3.835 \mathrm{Mcal} / \mathrm{ha})$. Em média, a participação da casca na quantidade de calor dessas espécies foi de $29 \%$. Estas doze espécies juntas foram responsáveis por $53.906 \mathrm{Mcal} / \mathrm{ha}$ ou $90 \%$ da energia disponibilizada. A escolha de espécies para a produção de energia na forma de calor com o objetivo de cocção de alimento deve ter como base duas características principais: maior produção de biomassa seca e maior massa específica, desde que não muito elevada, pois madeiras muito duras têm dificuldade em queimar, principalmente nos fogões rústicos usados no meio rural. Aliado a essas características deve-se levar em conta o poder calorífico superior da madeira. Essas características em conjunto propiciam grandes produções de energia na forma de calor. Outra característica que pode ajudar nessa escolha é o teor de carbono fixo, pois quanto maior o teor maior o tempo de residência do combustível no aparelho.

\section{CONCLUSÕES}

Em princípio a biomassa de qualquer uma das 47 espécies encontradas na área de estudo pode ser utilizada para geração de calor. No entanto, se o objetivo é otimizar a produção de calor algumas caracterísiticas devem ser observadas, tais como: produção de massa seca, massa específica, teor de carbono fixo e poder calorífico. Considerando tais características, dez espécies são responsáveis por $90 \%$ da energia na forma de calor disponível na área. Essas espécies estariam aptas a serem usadas num possível plano de produção sustentada de biomassa para geração de calor. Três espécies destacam-se, pois totalizaram $27.437 \mathrm{Mcal} / \mathrm{ha}$ e foram responsáveis por $45,72 \%$ de toda energia disponibilizada na área. São elas a Dalbergia miscolobium, o Pterodon pubescens e o Sclerolobium paniculatum que associam elevada produção de massa seca, maiores massas específicas, poderes caloríficos acima da média da área e valores médios para carbono fixo.

\section{REFERÊNCIAS BIBLIOGRÁFICAS}

ABNT. NBR 8633/84: Rio de Janeiro, 1994. 13 p.

AROUCA, M. C.; GOMES, F. B. M.; ROSA, L. P. Estrutura da demanda de energia no setor residencial no Brasil e uma avaliação da energia para cocção de alimentos: área interdisciplinar de energia COPPE/UFRJ. Rio de Janeiro, 1983. 36p. (Série de publicações - AIE 002/83).

ASTM. American Society for Testing and Materials. D 1762-64 (Reapproved 1977). 578 p.

BRASIL. Ministério de Minas e Energia. Balanço energético nacional. Brasília, 2000. 154p.

BRITO, J.O.; BARRICHELO, L.E.G.Aspectos técnicos da utilização da madeira e carvão vegetal como combustíveis. In: SEMINÁRIO DE ABASTECIMENTO ENERGÉTICO INDUSTRIAL COM RECURSOS FLORESTAIS, 2., 1982, São Paulo. São Paulo, 1982. p. 101-137. 
BRITO, J.O.; BARRICHELO, L.E.G. Características do eucalipto como combustível: análise química imediata da madeira e da casca. IPEF, v.16, p.63-78, 1978.

BRITO, J.O.; DEGLISE, X. States and potencial of using wood for energy in Brazil. Revue Forestière Française, Paris, n.6, p.175-79, 1991.

BURGER, L. M.; RICHTER, H. G. Anatomia da madeira. São Paulo: Nobel, 1991. 154 p.

CASTILLO, M.U. Determinacion del poder calorifico de 20 especies forestales de la Amazonia peruana. Revista Florestal do Peru, v.12, n.1-2, p.98-117, 1984.

CUNHA, M.P.S.C.; PONTES, C.L.F.; CRUZ, I.A.; CABRAL, M.T.F.D.; NETO, Z.B.C.; BARBOSA, A.P. Estudo químico de 55 espécies lenhosas para geração de energia em caldeiras. In: ENCONTRO ENCONTRO BRASILEIRO EM MADEIRAS E EM ESTRUTURAS DE MADEIRA, 3., 1989, São Carlos. Anais... São Carlos, 1989. v. 2, p.93-120.

EMBRAPA. Levantamento de reconhecimento dos solos do Distrito Federal. Serviço Nacional de Levantamento e Conservação dos Solos, 1978. Boletim Técnico, 53.

FOELKEL, C.E.B.; BRASIL, M.A.M.; BARRICHELO, L.E.G. Métodos para determinação da densidade básica de cavacos para coníferas e folhosas. IPEF, n.2/3, p.65-74, 1971.

FURLEY, P.A.; RATTER, J.A. An assessment of soil constraits on the distribution of plant communities at Fazenda Água Limpa, Brasília, $D F$. In: INTERNATIONAL SYMPOSIUM ON BIOLOGY OF CERRADO PLANTS, 1990, Campinas. Anais ... Campinas, 1990.

GOLDEMBERG, J. Energia, meio ambiente \& desenvolvimento. São Paulo: Ed. Universidade de São Paulo, 1998. 125p.

GRASSI, G.; PALZ, W. O futuro da biomassa na União Européia. Álcool. \& Açúcar, n.76, p.28-34, 1988.

HALL, D. O. Biomass energy. Energy Policy - Renewables series, p. 711-737, Oct., 1991.

JARA, E.R.P. O poder calorífico de algumas madeiras que ocorrem no Brasil. IPT - Comunicação Técnica, n.1797, p. 1-6, 1797.

MARABOTO, M.T.; CUNHA, M.P.S.C.; PONTES, C.L.F.; CRUZ, I.A.; NETO, Z.B.C. Poder calorífico e pirólise de dez espécies florestais da Amazônia Brasileira-Peruana. In: ENCONTRO BRASILEIRO EM MADEIRAS E EM ESTRUTURAS DE MADEIRA, 3., 1989, São Carlos. Anais... São Carlos, 1989. v.2, p.6-28.

MATA, H. T. C.; SOUZA, A. L. Estimativa do consumo residencial de lenha num distrito do Estado de Minas Gerais, Brasil. Rev. Árvore, Viçosa, v.24, n.1, p.63-71, 2000.

OLIVEIRA, A.D.; LEITE, A.P.; BOTELHO, S.A.; SCOLFORO, J.R.S. Avaliação econômica da vegetação de cerrado submetido a diferentes regimes de manejo e de povoamentos de eucalipto plantado em monocultivo. CERNE, v.4, n.1, p.34-56, 1998.

SILVA, F.C. Compartilhamento de nutrientes em diferentes componentes da biomassa a érea em espécies arbóreas de um cerrado. 1990. 80p. Dissertação (Mestrado em Ecologia) - Instituto de Ciências Biológicas, Departamento de Ecologia, Universidade de Bras ília, Brasília.

SILVA, M.A. Mudanças na composição florística e estrutura de um cerrado sensu stricto em um período de 12 anos (1985-1997), na Fazenda Água Limpa (FAL) - Distrito Federal. Brasília, 1999. 62p. Dissertação (Mestrado em Ciências Florestais) - Faculdade de Tecnologia, Departamento de Engenharia Florestal, Universidade de Brasília, Brasília.

VITAL, B.R. Métodos de determinação da densidade da madeira. Viçosa: SIF, 1984. 21p. (Boletim Técnico, 2).

VITAL,B.R. Tecnologia da Madeira: métodos para determinação do teor de umidade da madeira. Viçosa: SIF, 1997. 33p. (Boletim Técnico, 13). 